\title{
Estado de conservación de Chiasognathus granti Stephens 1831 (Coleoptera: Lucanidae) en Chile
}

\author{
Conservation status of Chiasognathus granti Stephens 1831 (Coleoptera: Lucanidae) \\ in Chile
}

OLIVIA E. VERGARA* \& VIVIANE JEREZ

Departamento de Zoología, Facultad de Ciencias Naturales y Oceanográficas, Universidad de Concepción. Casilla 160-C

*Autor correspondiente: olvergar@udec.cl

\begin{abstract}
RESUMEN
Las estrategias de conservación en Chile han analizado la diversidad de nuestro país en base a información de especies grandes, carismáticas, paraguas y poco abundantes. Actualmente, nuestro interés ha estado dirigido a analizar la información de invertebrados para definir áreas de endemismo, áreas prioritarias para conservación y áreas naturales importantes desde el punto de vista filogenético. El lucánido Chiasognathus granti es una especie emblemática para la entomología chilena. Este trabajo es pionero en evaluar el estado de conservación de esta especie en Chile, para lo cual fueron calculados los índices de prioridad de conservación (SUMIN y $\mathrm{CPI}$ ) en base a literatura especializada, colecciones de referencia y prospecciones en terreno. $C$. granti es considerada una especie Rara, de acuerdo al índice de SUMIN y Vulnerable, de acuerdo al índice CPI. Nosotros concluimos que $C$. granti puede ser considerada Vulnerable en Chile con una alta probabilidad de extinción, hecho que podría afectar la sobrevivencia de las poblaciones presentes en Argentina.
\end{abstract}

Palabras clave: Bosque Valdiviano, Chiasognathus, índices de conservación.

\begin{abstract}
Based on information of large body size, charismatic, umbrella and Rare species, the Conservation strategies have analyzed the diversity of Chile. Define endemism areas, priorities conservation areas and geographic areas with high phylogenetic value has been the aims of our studies in beetles. An emblematic species for the Chilean entomologic community is the stag beetle $C$. granti. We assign priorities for the conservation of $C$. granti through conservation priority indexes (SUMIN and CPI) based on literature, reference collection and field studies. $C$. granti can be considered Rare by SUMIN index and Vulnerable by CPI index. We concluded that $C$. granti is Vulnerable with a high probability of extinction in Chile; this situation could affect the survival of populations in Argentina.
\end{abstract}

Key words: Chiasognathus, conservation index, Valdivian forest.

\section{INTRODUCCIÓN}

La biología de la conservación tiene como objetivos informar sobre la toma de decisiones sobre la conservación de la biodiversidad preservando muestras representativas de ecosistemas y manteniendo la viabilidad de sus poblaciones (Whittaker et al. 2005). A través de la aplicación de métodos científicos, intenta comprender la crisis ambiental causada por la actividad antrópica (Rozzi et al. 2001). ¿Qué proteger?, ¿por qué proteger? y ¿cómo proteger? son todas preguntas clave para definir estrategias de conservación en el mundo (Primack et al. 2001, Lichtschein et al. 2006 ${ }^{1}$ ).

Los estudios de conservación de la diversidad en un lugar determinado, pueden enfocarse hacia los ecosistemas y comunidades a

1 LICHTSCHEIN V, L ARENAS \& M TONELLI (2006) Coordinación de conservación de la Biodiversidad. II Congreso Nacional de la Conservación de la Biodiversidad, Ciudad de Buenos Aires, Argentina. 
través de los Sitios Prioritarios para la Conservación (Arzamendia \& Giraudo 2004) en áreas de alta diversidad y amenaza (Hotspots) (Myers et al. 2000, Redak 2000), sitios de transición (Morrone 2006) o de alta complementariedad (Bossart \& Carlton 2002) y centros o áreas de endemismos (Rojas 1992, Roig-Juñent et al. 2002, Domínguez et al. 2006) todos los cuales pueden ayudar a establecer Áreas Silvestres Protegidas (ASP) (Primack et al. 2001). Por otra parte, si los estudios están dirigidos a determinar el estado de conservación de las especies, el criterio más utilizado es el de rareza que permite estimar el riesgo de extinción de los taxa involucrados (Primack et al. 2001, De Grammont \& Cuarón 2006). Así, las especies raras, según Rabinowitz (1981) son definidas como "especies poco abundantes, con rangos de distribución restringidos y estrecha especificidad de hábitat" (sensu Thomas \& Mallorie 1985, Eyre \& Rushton 1989).

En relación a los sistemas de clasificación de especies con problemas de conservación, destaca a nivel mundial la Lista Roja de especies amenazadas (IUCN 2001), principalmente de vertebrados y para Chile el Reglamento para la Clasificación de especies de Flora y Fauna Silvestre (CONAMA 2005) dirigido a plantas vasculares y vertebrados.

Las especies pueden ser definidas en alguna prioridad de conservación sobre la base de diferentes criterios: singularidad (especies únicas), distribución geográfica restringida (endemismos) (Primack et al 2001), riesgo de extinción (Estado de Conservación) y factibilidad de recuperación y/o manejo. Otros criterios a considerar son los efectos adicionales que conlleva su conservación directa, como son la conservación de otras especies, de su hábitat, conservación de especies "paraguas", si son culturalmente especies carismáticas o si presentan interés económico (Fleishman et al. 2000, Primack et al. 2001).

Entre los organismos más utilizados en programas de conservación en Chile, resaltan las plantas (Squeo et al. 2001), los mamíferos (Povilitis 1998, Cofré \& Marquet 1999), las aves (Glade 1988, Jiménez 1999), los reptiles (en prensa CONAMA) y en menor medida los anfibios (Díaz-Páez \& Ortiz 2003). La mayoría de estos taxa corresponden a especies carismáticas $\mathrm{y} / \mathrm{o}$ paraguas, ecológica y biológicamente conocidas (Povilitis 1998, Arzamendia \& Giraudo 2004) y que con variadas metodologías de muestreo (trampas, avistamientos, fecas, huellas, etc.) son relativamente más factibles de estudiar. Muchas de ellas son además, especies importantes para el ecoturismo, situación que explica la tendencia a incluir en las listas de especies raras o amenazadas, a especies bien estudiadas y de gran tamaño (Christie 1984, Schwartz 1999, Arroyo et al. 2008).

Entre las estrategias de conservación dirigidas a insectos en el mundo, están por ejemplo la elaboración de índices de rareza para coleópteros acuáticos en Gran Bretaña (Foster 1985 sensu Eyre \& Rushton 1989), la categorización de especies de insectos en Estados Unidos (Bossart \& Carlton 2002) y el Libro Rojo de Invertebrados en España (Verdú \& Galante 2005). En Chile, aún no existe información respecto a la generación de propuestas de conservación para invertebrados; aunque paradójicamente muchos autores señalan su utilidad para ser empleados en conservación sin desarrollar hasta el momento iniciativas concretas para este efecto. En consecuencia y aunque la documentación de especies de invertebrados amenazadas es una tarea urgente a realizar, ha sido muy poco explorada a nivel mundial (Sanways 2001).

Para categorizar especies en algún estado de conservación, han sido utilizados diversos índices entre los que destacan los índices de prioridad de conservación SUMIN y CPI basados en aspectos morfológicos, distribucionales y ecológicos de las especies (Reca et al. 1994, Úbeda et al. 1994, Diaz-Páez \& Ortiz 2003) y el cálculo de rareza que estima el riesgo de extinción (Cofré \& Marquet 1999). Aunque es factible catalogar en algún estado o prioridad de conservación a insectos culturalmente simbólicos y/o carismáticos para la comunidad científica, organizaciones gubernamentales, no gubernamentales (ONG) y público en general, estos índices no han sido utilizados en entomología en Chile.

La cantabria Chiasognathus granti Stephens 1931, lucánido endémico del sur de Sudamérica, se caracteriza por presentar una llamativa morfología, esplendor y colorido (Numhauser 1981); en Chile habita bosques de Nothofagus propios de la selva valdiviana o bien en sectores que históricamente estuvieron 
cubiertos por dicha formación y que actualmente están destinados a uso agrícola y/o forestal (Vergara \& Jerez 2008²). En consecuencia, los objetivos de este trabajo son establecer criterios operacionales que permitan determinar la prioridad de conservación y estado de conservación de $C$. granti en Chile, en base a aspectos biológicos, ecológicos y biogeográficos. La aplicación y comparación de distintos índices, permitirá determinar cuál es el más apropiado para ser aplicado en el futuro a coleópteros en particular e insectos en general.

\section{MÉTODOS}

Para determinar el estado del conocimiento biológico, ecológico y biogeográfico de la cantabria o ciervo volante, recopilamos y analizamos la información obtenida a través de una exhaustiva revisión bibliográfica (Revista Chilena de Historia Natural, Revista Chilena de Entomología, Acta Entomológica Chilena, Boletín de la Sociedad de Biología de Concepción, Revista Bosque, Revista Universitaria, Academia Chilena de Ciencias Naturales, Notas del Museo Universidad Nacional de Eva Perón, Transactions of the Entomological Society of London, Records of the Dominion Museum, New Zealand Journal of Science, Proceedings of the Royal Entomological Society of London, Boletín del Museo Nacional de Chile, Elytron, entre otras), de bases de datos de registros de especies y colecciones de referencia (Museo Nacional de Historia Natural, Santiago (MNHN), Museo Nacional de Historia Natural, Concepción (MNHNC), Museo de Zoología, Universidad de Concepción (UCCC), Instituto de Entomología de la Universidad Metropolitana de Ciencias de la Educación (UMCE), Instituto Argentino de Investigaciones de Zonas Áridas (IADIZA), Argentina, Division of Insect, Zoology, Field Museum, Estados Unidos y Colecciones particulares). Adicionalmente, efectuamos prospecciones en terreno, abarcando el rango

VERGARA O \& V JEREZ (2008) Rangos de distribución latitudinal de Chiasognathus granti Stephens 1831 en Chile. VII Congreso Argentino de Entomología, octubre, Córdoba, Argentina. de distribución de la especie en sectores no protegidos y en Áreas Silvestres Protegidas por el Estado de Chile como son los Parques Nacionales Villarrica $\left(39^{\circ} 15^{\prime} \mathrm{S}\right.$ y $\left.72^{\circ} 12^{\prime} \mathrm{O}\right)$, Vicente Pérez Rosales $\left(41^{\circ} 11^{\prime}\right.$ 'S y $72^{\circ} 32^{\prime}$ O O), Puyehue $\left(40^{\circ} 39^{\prime}\right.$ 'S y $72^{\circ} 09^{\prime}$ O) y Jeinimani (463' $\mathrm{S}$ y $\left.71^{\circ} 40^{\prime} \mathrm{O}\right)$, las Reservas Nacionales Malalcahuello (38 $43^{\prime}$ S y $77^{\circ} 34^{\prime}$ O), Río Simpson $\left(45^{\circ} 27^{\prime} \mathrm{S}\right.$ y $\left.72^{\circ} 22^{\prime} \mathrm{O}\right)$, Mañihuales $\left(45^{\circ} 21^{\prime} \mathrm{S}\right.$ y $\left.72^{\circ} 27^{\prime} \mathrm{O}\right)$ y Cerro Castillo (45 $56^{\circ}$ S y $72^{\circ} 58^{\prime}$ O), el Parque Privado Oncol (39 $41^{\circ}$ ' S y $73^{\circ} 19^{\prime}$ O) y el Monumento Nacional Contulmo (38 $00^{\prime}$ 'S y $73^{\circ} 13^{\prime} \mathrm{O}$ ). Los puntos de colecta en terreno fueron georreferenciados $\mathrm{y}$ son mostrados en la Fig. 1.

Para establecer los estados y prioridades de conservación de la cantabria, aplicamos los índices de prioridad de conservación de SUMIN (Reca et al. 1994) y CPI (Cofré \& Marquet 1999). La Tabla 1 entrega un resumen de las características de ambos índices. Para cada índice y variable, elegimos, rechazamos y/o modificamos variables que son factibles de ser aplicadas para coleópteros en general y para $C$. granti en particular.

\section{Índices de prioridad de conservación}

Los índices de conservación SUMIN y CPI fueron evaluados para $C$. granti en base a las siguientes sumatorias:

$$
\text { SUMIN }=\sum_{\mathrm{j}=1}^{12} \mathrm{Vij} \quad \mathrm{CPIi}=\sum_{\mathrm{j}=1}^{9} \mathrm{Vij}
$$

En donde $\mathrm{V}$ representa cada una de las variables analizadas que son descritas a continuación:

Variables utilizadas para SUMIN:

Distribución continental (DICON): de acuerdo a Reca et al. (1994) donde se establece una proporción del territorio continental habitado por la especie.

Distribución nacional (DINAC): el territorio chileno fue dividido en cuartos obteniéndose un valor porcentual aproximado de la distribución de la especie.

Amplitud del uso de hábitat (AUHA): considera si la especie utiliza uno o más hábitats en estado adulto.

Amplitud en el uso del espacio vertical (AUEVE): considera si la especie en estado 
adulto, utiliza uno o más estratos para efectos de reproducción y/o alimentación; se considera el tipo de actividad biológica en la cual los individuos utilizan el menor número de estratos. Los estratos de vegetación, fueron clasificados en base a la propuesta de estratificación de Smith \& Smith (2001): zonas de copas, zona inferior de copas, sotobosque, estrato arbustivo, estrato herbáceo y capa de materia orgánica.

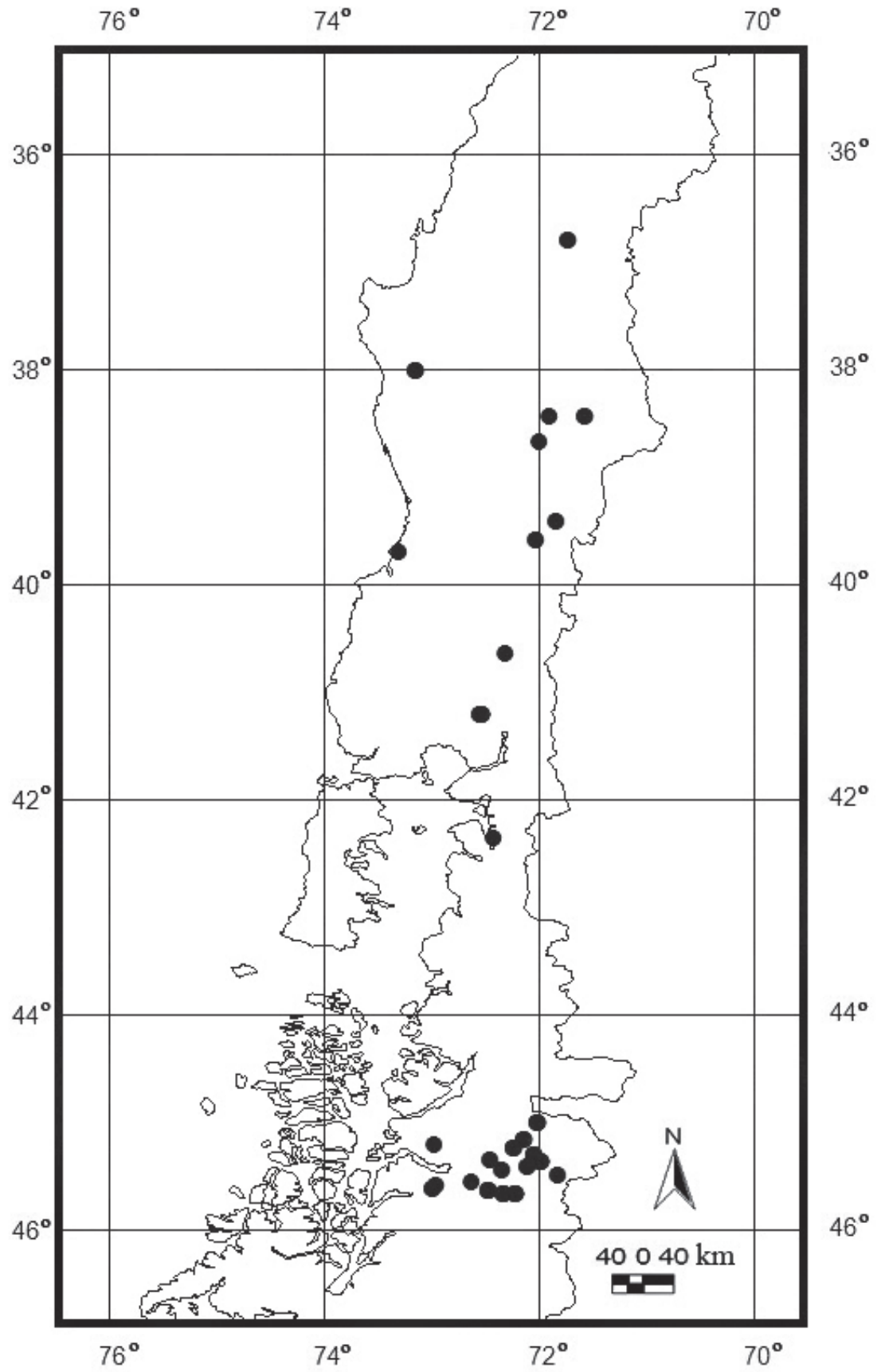

Fig. 1: Prospecciones en terreno en Chile para Chiasognathus granti entre 2006-2008. Los puntos negros indican localidades de colecta.

Field studies from Chile for C. granti between 2006-2008 years. Black point shows localities records. 
TABLA 1

Comparación entre índices de prioridad de conservación utilizados.

Comparison between conservation priorities indexes.

\begin{tabular}{lcc}
\hline Índices / características & SUMIN & CPI $^{1}$ \\
\hline Variables & Al menos 12 & Al menos 9 \\
Fórmula & Suma de todas las variables & Suma de todas las variables \\
Valores total & $0-30$ & $0-27$ \\
Valor para c/variable & $0-5$ & $0-3$ \\
Algunas de sus variables & Distribución nacional, Amplitud trófica & Promedio de abundancia Local, \\
singularidad taxonómica
\end{tabular}

1 Índice de Prioridad de Conservación

Para la variable "tamaño corporal" hemos considerado por separado la longitud promedio del macho y de la hembra debido al gran dimorfismo sexual que presenta esta especie.

Tamaño corporal del macho (TAMm): Variable modificada de Reca et al. (1994) y redefinida para $C$. granti como la longitud corporal (sin considerar antenas ni mandíbulas). Calculamos esta variable comparándola con la información de la longitud corporal para machos de las especies de Lucanidae registradas para Chile. Los rangos de longitud se calcularon de acuerdo a clases de tamaño de acuerdo a las siguientes fórmulas:

$$
\begin{gathered}
\text { Amplitud }=\frac{\text { Rango }}{\text { Intervalo }} \\
\text { Intervalo }=1+3,3 \log (n)
\end{gathered}
$$

Tamaño corporal de la hembra (TAMh): Modificada de Reca et al. (1994) al igual que en la variable anterior.

Potencial reproductivo (POTRE): número de huevos por ovipostura conocidos para las especies.

Amplitud trófica del adulto (AMPRO). Determinada de acuerdo a su estrategia alimenticia.

Abundancia (ABUND): tamaño poblacional estimado dentro del país, ponderado mediante una escala relativa. Una especie es abundante cuando sus grupos poblacionales presentan gran número de individuos. Común cuando su presencia es continua, sin importar el número de individuos.
Escasa, con grupos poblacionales con bajo número de individuos. Rara, presencia discontinua en el área de distribución y con bajo número de individuos. Cuando exista dudas entre dos ítemes se considera el peor escenario posible.

Singularidad taxonómica (SINTA): si la especie es considerada única.

Singularidad (SING): estimada de las características particulares que afectan la sobrevivencia de las especies o que son relevantes para su conservación como su endemismo como por ejemplo.

Acción extractiva (ACEXT): referida a si existe remoción de individuos en forma antrópica.

Grado de protección (PROT): presencia de la especie en áreas naturales en las que efectivamente se efectúan tareas de control a través del Estado, mediante la Corporación Nacional Forestal (CONAF) u organismos privados.

\section{Variables utilizadas para CPI:}

Especificidad de hábitat (HSP): número de ecorregiones en las que la especie ha sido encontrada. Para este caso, consideramos las ecorregiones propuestas por Cofré \& Marquet (1999).

Distribución geográfica de la especie en Chile (GD): Variable modificada de Cofré \& Marquet (1999) en la que consideramos la superficie regional en que la especie habita (sensu Navarrete 1992) y su representación en $\mathrm{km}^{2}$ para un valor de $756,253 \mathrm{~km}^{2}$ como superficie total para Chile. 
Abundancia (AB): considerada como el promedio de abundancia local (ind $\mathrm{km}^{-2}$ ).

Endemismo (END): basado en el número de países donde la especie está presente.

Singularidad taxonómica (TSING): basada en el nivel monotípico de familia y/o género.

Masa corporal (BM-m): modificada de Cofré \& Marquet (1999) y reemplazada por el largo corporal promedio del macho y cuyos valores fluctúan entre 22 y $80 \mathrm{~mm}$.

Presencia de la especie en otras listas de conservación (POL).

Efectos de actividades humanas (HE): Incidencia directa de actividades humanas que afectan a la especie.

Grado de prospección (DP): basado en el porcentaje de área de una ecorregión (definida por Luebert \& Pliscoff [2006]), que es habitada por la especie y que coincide con un área protegida.

Para establecer los estados de conservación y prioridades de conservación de $C$. granti en Chile, los valores totales obtenidos de la suma de las variables consideradas para cada índice fueron distribuidas en cinco rangos que corresponden a cinco categorías de conservación. Para SUMIN: En Peligro, Vulnerable, Rara, Insuficientemente Conocida y Fuera de Peligro. Para CPI: Crítica, En Peligro, Vulnerable, Frágil y Sin Prioridad Inmediata (Tabla 2).

\section{RESULTADOS}

La Tabla 1 entrega un resumen del número de variables que deben ser consideradas para establecer prioridades de conservación y el rango de valores que cada variable puede tener.
En este estudio, consideramos 13 variables para el índice de prioridad de conservación SUMIN, con valores que fluctúan entre 0 y 5 . Para el índice CPI consideramos 9 variables, con valores que fluctúan entre 0 y 3 . Los índices de prioridad de conservación SUMIN y CPI aplicados a $C$. granti, las variables analizadas y los valores resultantes para cada variable son mostrados en las Tablas 3 y 4 . A continuación son descritas las variables analizadas y/o modificadas en función del conocimiento biológico, ecológico y biogeográfico que disponemos para C. granti.

\section{Variables modificadas del índice de SUMIN:}

Amplitud de uso de hábitat (AUHA): hemos comprobado que $C$. granti habita principalmente en ambientes de bosque adulto con presencia de Nothofagus (en dinámica post-fuego) y ambientes de praderas rodeados por bosques de Nothofagus. Esto ha sido comprobado en la Región de Aysén a través de prospecciones efectuadas durante los años 2006-2007.

Amplitud de espacio vertical (AUEVE): Según Reca et al. (1994), debemos utilizar la actividad ecológica (en este caso la reproducción), en la cual la especie utilice el menor número de estratos. Los adultos de $C$. granti utilizan al parecer entre dos a tres estratos para su reproducción, ya sea en la zona inferior de las copas, sotobosque y capa de materia orgánica.

Tamaño corporal de machos (TAMm): para las especies de Lucanidae en Chile hemos analizado la longitud corporal de los machos concluyendo que esta varía entre 10 y $80 \mathrm{~mm}$. Para el caso particular de C. granti hemos

TABLA 2

Categorización de los estados de conservación y valores para los índices SUMIN y CPI.

Categorization of Conservation Status and SUMIN and CPI indexes value.

\begin{tabular}{lccc}
\hline SUMIN & \multicolumn{2}{c}{ CPI } & Valores \\
\cline { 2 - 4 } Categoría & Valores & Categoría & $0-3$ \\
\hline Fuera de Peligro & $0-4$ & Sin Prioridad Inmediata & $4-8$ \\
Insuficientemente Conocida & $5-10$ & Frágil & $9-13$ \\
Rara & $11-16$ & Vulnerable & $14-18$ \\
Vulnerable & $17-22$ & En Peligro & $19-21$ \\
En peligro & $23-29$ & Crítica & \\
\hline
\end{tabular}


utilizado valores de longitud corporal de machos recolectados en la Región de Aysén que varían entre 22 y $80 \mathrm{~mm}$ con un promedio de $30.1 \mathrm{~mm}$ y rangos entre $10-28.38 \mathrm{~mm}, 28.39-46.77 \mathrm{~mm}$ y 46.78-65.17 mm de longitud.

Tamaño corporal de hembras (TAMh): para las especies de Lucanidae en Chile, hemos analizado la longitud corporal de la hembra la que varía entre 5 y $39 \mathrm{~mm}$. En este estudio hemos utilizado valores de longitud corporal de hembras recolectadas en la Región de Aysén, con una longitud promedio de $28 \mathrm{~mm}$ y rangos entre 5-17.59 mm, 17.6-30.19 $\mathrm{mm}$ y 30.2-42.79 mm de longitud.

Potencial reproductivo (POTRE): esta variable no fue utilizada, ya que es desconocido el número de huevos aproximado por postura en esta especie.

Amplitud trófica del adulto (AMPRO): Según nuestro conocimiento de la historia natural de esta especie, los adultos de $C$. granti liban la savia exudada de Nothofagus betuloides (Mirbel) Oersted, Nothofagus nitida (Phil.) Krassen y Weinmannia trichosperma Cav. Sus larvas son radicícolas en praderas donde dominan Dactylis glomerata L. y Trifolium repens $\mathrm{L}$. En este sentido, $C$. granti correspondería a un herbívoro generalista.

Abundancia (ABUND): Hemos estudiado la distribución de las poblaciones de $C$. granti en Chile, concluyendo que no presentan un elevado número de individuos y en la mayor parte del país su distribución es discontinua y agregada, por tanto, actualmente no existe certeza si $C$. granti es escasa o rara. Al considerar el peor escenario posible de acuerdo a uno de los principios en gestión ambiental, $C$. granti presentaría para Chile, una abundancia rara.

Grado de protección (PROT): C. granti está protegida en Chile, en dos unidades de conservación efectiva: la Reserva Nacional Simpson $\left(45^{\circ} 27^{\prime}\right.$ S y $72^{\circ} 22^{\prime}$ O) y Reserva Nacional Puyehue (40³9' S y $72^{\circ} 09^{\prime}$ O) (Fig. 1). Hemos descartado la unidad de conservación Parque Nacional Vicente Pérez Rosales, dado su alto nivel de antropización y la falta de información para definir si esta especie está o no sometida a alguna protección dentro del Parque.

Variables modificadas del índice de CPI:

Especificidad de Hábitat (HSP): definida como el número de ecorregiones donde la especie ha sido encontrada (sensu Cofré \& Marquet [1999]).

Distribución geográfica de la especie en Chile (GD): representada en $\mathrm{km}^{2}$ para 756,253 $\mathrm{km}^{2}$ como superficie total de Chile. Consideramos la suma de las superficies de cada región en que la especie ha sido registrada (sensu Navarrete [1992]).

Abundancia (AB): considerada como el promedio de abundancia local (individuos $\mathrm{km}^{-2}$ ). Esta variable no fue considerada, ya que no existe una estandarización confiable para definir la densidad promedio de la especie en Chile.

Endemismo (END): la información actual sobre esta especie no define con precisión, alguna característica importante que nos permita delimitar biogeográficamente las ecorregiones a nivel continental en que esta especie está presente, por lo que esta variable fue basada en el número de países donde está presente.

Singularidad taxonómica (TSING): basado en el nivel monotípico a nivel de familia y género.

Masa corporal (BM-m): esta variable fue modificada de Cofré \& Marquet (1999) y reemplazada por el largo corporal promedio del macho. Los valores estándares para las especies de Lucanidae en Chile varían desde 22 a 80 $\mathrm{mm}$. Como necesitamos cuatro categorías, definimos para $C$. granti rangos entre $\geq 28.38$ $\mathrm{mm} ; 28.39-46.77 \mathrm{~mm}$; 46.78-65.17 mm; 65.18 $\leq \mathrm{mm}$.

Presencia en otras listas (POL): esta variable no fue considerada, ya que el estado de conservación de $C$. granti no ha sido evaluado con anterioridad.

Efectos de actividades humanas (HE): evaluada de acuerdo a información recopilada de literatura y datos de prospecciones en terreno.

Grado de prospección (DP): basado en el porcentaje de área de una ecorregión (Luebert \& Pliscoff 2006) habitada por la especie y que coincide con un área protegida.

\section{Categorización del estado de conservación:}

La Tabla 2 muestra la categorización de estados de conservación establecidos para ambos índices a partir de este estudio. En base a los valores obtenidos de la suma de las variables y criterios considerados en la Tabla 3 para 
TABLA 3

Chiasognathus granti. Criterio para cada variable del Índice de SUMIN. Modificado de Reca et al. (1994) para el análisis de insectos. * Muestra el valor que resulta para cada variable.

Chiasognathus granti. Test for SUMIN Index. Modified from Reca et al (1994) for insect analysis. *Show the variable results.

\begin{tabular}{|c|c|c|c|c|c|c|c|}
\hline Variable & Valor 0 & Valor 1 & Valor 2 & Valor 3 & Valor 4 & Valor 5 & Valores \\
\hline DICON & $\begin{array}{l}\text { Todo el continente } \\
\text { o en su mayor parte }\end{array}$ & $\begin{array}{c}\text { Aproximadamente } \\
\text { la mitad del } \\
\text { continente }\end{array}$ & $\begin{array}{c}\text { Menos de la } \\
\text { mitad del } \\
\text { continente }\end{array}$ & Restringida* & & & 3 \\
\hline DINAC & $\begin{array}{l}\text { Todo el país o } \\
\text { su mayor parte }\end{array}$ & $\begin{array}{l}\text { Aproximadamente } \\
\text { la mitad del país }\end{array}$ & $\begin{array}{l}\text { Menos de la } \\
\text { mitad del país* }\end{array}$ & Restringida & $\begin{array}{l}\text { Muy localizada } \\
\text { o endemismo }\end{array}$ & Microendemismo & 2 \\
\hline AUHA & $\begin{array}{l}\text { Puede utilizar } 4 \text { ó } \\
\text { más ambientes }\end{array}$ & $\begin{array}{l}\text { Puede utilizar } \\
2 \text { ó } 3 \text { ambientes }\end{array}$ & $\begin{array}{l}\text { Puede utilizar } \\
\text { solo } 1 \text { ambiente } \\
\text { o necesitar más } \\
\text { de uno* }\end{array}$ & & & & 2 \\
\hline AUEVE & $\begin{array}{l}\text { Puede utilizar } 4 \\
\text { ó más estratos }\end{array}$ & $\begin{array}{c}\text { Puede utilizar } \\
\text { de } 2 \text { a } 3 \text { estratos* }\end{array}$ & $\begin{array}{l}\text { Puede utilizar } \\
\text { solo un } \\
\text { estrato, o } \\
\text { necesitar más } \\
\text { de uno }\end{array}$ & & & & 1 \\
\hline TAMm & $\begin{array}{c}\text { Entre } 10-28.38 \\
\text { mm de largo }\end{array}$ & $\begin{array}{l}\text { Entre } 28.39- \\
46.77 \mathrm{~mm} \text { de } \\
\quad \text { largo* }\end{array}$ & $\begin{array}{c}\text { Entre } 46.78 \\
-65.17 \mathrm{~mm} \mathrm{de} \\
\operatorname{largo}\end{array}$ & & & & 1 \\
\hline TAMh & $\begin{array}{c}\text { Entre } 5-17.59 \\
\text { mm de largo }\end{array}$ & $\begin{array}{l}\text { Entre } 17.6- \\
30.19 \mathrm{~mm} \\
\text { de } \operatorname{largo*}\end{array}$ & $\begin{array}{l}\text { Entre } 30.2- \\
42.79 \mathrm{~mm} \mathrm{de} \\
\text { largo }\end{array}$ & & & & 1 \\
\hline POTRE & > a 1000 huevos & 100 - 999 huevos & $<99$ huevos & & & & $\mathrm{NE}$ \\
\hline AMTRO & $\begin{array}{l}\text { Omnívoras y } \\
\text { herbívoros } \\
\text { generalistas* }\end{array}$ & $\begin{array}{l}\text { Herbívora } \\
\text { especialista } \\
\text { detritívoro, } \\
\text { carnívoro } \\
\text { generalista } \\
\text { y carroñero }\end{array}$ & $\begin{array}{l}\text { Carnívoro } \\
\text { especialista }\end{array}$ & & & & 0 \\
\hline ABUND & Escasa & Rara o muy rara* & & & & & 1 \\
\hline SINTA & Ausencia* & $\begin{array}{l}\text { Pertenece } \\
\text { a un género } \\
\text { monotípico }\end{array}$ & $\begin{array}{l}\text { Pertenece a } \\
\text { familia o } \\
\text { taxón de } \\
\text { nivel superior } \\
\text { monotípico y } \\
\text { monoespecífico }\end{array}$ & & & & 0 \\
\hline SING & Ausencia* & Presencia & & & & & 0 \\
\hline ACEXT & No hay & $\begin{array}{c}\text { Por ser } \\
\text { considerados } \\
\text { plagas o } \\
\text { perjudiciales, } \\
\text { caza por temor }\end{array}$ & $\begin{array}{l}\text { Caza por control, } \\
\text { explotación } \\
\text { comercial a } \\
\text { pequeña escala, } \\
\text { o por deporte }\end{array}$ & $\begin{array}{l}\text { Extracción por } \\
\text { dos o más de } \\
\text { los motivos } \\
\text { anteriores* }\end{array}$ & $\begin{array}{c}\text { Explotación } \\
\text { intensiva } \\
\text { para } \\
\text { comercialización }\end{array}$ & & 3 \\
\hline PROT & $\begin{array}{l}\text { Protegida por } 3 \\
\text { ó más unidades } \\
\text { de conservación }\end{array}$ & $\begin{array}{l}\text { Protegida por } 2 \\
\text { unidades de } \\
\text { conservación* }\end{array}$ & $\begin{array}{l}\text { Protegida por } \\
1 \text { unidad de } \\
\text { conservación }\end{array}$ & No protegida & & & 1 \\
\hline TOTAL & 0 & 5 & 4 & 6 & & & 15 \\
\hline
\end{tabular}


SUMIN y Tabla 4 para CPI, $C$. granti tiene un valor de 15 para SUMIN con el cual debe ser categorizada como Rara y de 11 para CPI que corresponde al estado de conservación Vulnerable.

\section{DISCUSIÓN}

Uno de los objetivos de la Estrategia Nacional de Biodiversidad (CONAMA 2003) y de acuerdo al Convenio sobre Diversidad Biológica, es categorizar las especies amenazadas de acuerdo a su probabilidad de extinción. Sin embargo, en Chile no existe una política clara en relación a categorizar especies de insectos amenazadas, probablemente debido a problemas metodológicos, limitaciones económicas (Reca et al. 1994) y/o taxonómicas asociadas a su alta diversidad (Refseth 1980, Simonetti 1997) o bien al poco interés o "simpatía" que la sociedad tiene en general sobre algunos insectos (Kellert 1993, Redak 2000, Bossart \& Carlton 2002).

C. granti, especie emblemática por su distribución sudamericana (endémica de la selva Valdiviana), relación trófica con ambientes de Nothofagus, taxonomía estable, e individuos fácilmente reconocibles, observables y atractivos para la comunidad, constituye un modelo adecuado para probar índices de prioridad de conservación y aplicables para futuros estudios de estrategias de conservación en Chile.

En el tema de la conservación de las especies, la legislación ambiental chilena es abundante pero fragmentada; al mismo tiempo carece de un sistema de organización y de una clara relación con otras categorías de conservación y finalmente no existe un cuerpo legal definido sobre la protección de la diversidad biológica (Iriarte et al. 1997; Pellet

TABLA 4

Chiasognathus granti. Criterio para cada variable del índice CPI. Modificado Cofré \& Marquet (1999) para C. granti. * Muestra el valor que resulta para cada variable.

Chiasognathus granti. Test for CPI index. Modified from Cofre \& Marquet (1999) for C. granti. *Show the variable results.

\begin{tabular}{|c|c|c|c|c|c|}
\hline Variables & Valor 0 & Valor 1 & Valor 2 & Valor 3 & Valores \\
\hline HSP & $\geq$ Cuatro ecorregiones & Tres ecorregiones* & Dos ecorregiones & Una ecorregión & 1 \\
\hline GD & $\geq 567,000 \mathrm{~km}^{2}$ & $378,000-567,000 \mathrm{~km}^{2}$ & $189,000-378,000 \mathrm{~km}^{2 *}$ & $\leq 189,000 \mathrm{~km}^{2}$ & 2 \\
\hline $\mathrm{AB}$ & $>1,000$ ind $\mathrm{km}^{-2}$ & $100-1,000$ ind $\mathrm{km}^{-2}$ & $10-100$ ind $\mathrm{km}^{-2}$ & $<10$ ind $\mathrm{km}^{-2}$ & $\mathrm{NE}$ \\
\hline END & Tres o más países & Dos países & Un país* & Solamente en Chile & 2 \\
\hline TSING & $\begin{array}{l}\text { Especie en género con } \\
\text { más de cuatro especies }\end{array}$ & $\begin{array}{c}\text { Especie en género con } \\
\text { dos, tres o cuatro } \\
\text { especies* }\end{array}$ & $\begin{array}{l}\text { Especie en género } \\
\text { monotípico. }\end{array}$ & $\begin{array}{l}\text { Especie en familia } \\
\text { monotípica }\end{array}$ & 1 \\
\hline $\mathrm{BM}(\mathrm{LC})$ & $<28.38 \mathrm{~mm}$ de largo & $\begin{array}{c}\text { Entre } 28.39-46.77 \mathrm{~mm} \\
\text { de largo* }\end{array}$ & $\begin{array}{l}\text { Entre } 46.78-65.17 \\
\text { mm de largo }\end{array}$ & $>65.17 \mathrm{~mm}$ de largo & 1 \\
\hline POL & $\begin{array}{l}\text { Ausente en lista } \\
\text { roja IUCN }\end{array}$ & $\begin{array}{c}\text { Rara, indeterminada } \\
\text { o inadecuadamente } \\
\text { conocida }\end{array}$ & Vulnerable & En peligro & NE \\
\hline $\mathrm{HE}$ & No conocido & $\begin{array}{c}\text { Afectada por } \\
\text { domesticación o } \\
\text { especies exóticas }\end{array}$ & $\begin{array}{c}\text { Control, subsistencia } \\
\text { o caza deportiva }\end{array}$ & $\begin{array}{l}\text { Destrucción de hábitat } \\
\text { y/o caza comercial* }\end{array}$ & 3 \\
\hline DP & $\begin{array}{c}\geq 50 \% \text { del área } \\
\text { de las ecorregiones } \\
\text { habitadas por la } \\
\text { especie es protegida }\end{array}$ & $50-30 \% *$ & $30-10 \%$ & $<10 \%$ & 1 \\
\hline Valores & 0 & 4 & 4 & 3 & 11 \\
\hline
\end{tabular}


et al. 2005). Por otra parte, no incluye evaluaciones del nivel de incerteza, ni consideraciones de la tolerancia al riesgo y no es aplicable a diferentes niveles taxonómicos ni a diferentes escalas geográficas (De Grammont \& Cuarón 2006). Se agrega a esto el error en la toma de decisiones por la falta de conocimiento taxonómico y biológico de las especies, deficiencias en el conocimiento del número total de especies, la falta de taxónomos y su impacto en la conservación de la biodiversidad (Simonetti 1997).

Esta propuesta del estado de conservación de $C$. granti resulta ser pionera en Chile en estudios entomológicos. Nuestro objetivo principal está enfocado a servir de base para futuros estudios en categorización de insectos y como una herramienta útil para la toma de decisiones en conservación a nivel nacional ya que antes de esta propuesta, la única información existente en cuanto al manejo o conservación de los tamaños poblacionales de la cantabria, se encuentra en el Reglamento de la Ley de Caza (Decreto Supremo No 05, SAG 1998) artículo 7 , donde se señala que "con el propósito de llevar estadísticas poblacionales, las personas naturales o jurídicas que capturen especies de invertebrados del género Chiasognathus (entre otros), se deberían inscribir e informar anualmente en dicho Servicio".

Los índices de conservación definidos para C. granti, permiten categorizarla como especie Rara según el índice de SUMIN y como Vulnerable según el índice de CPI. Ambos resultados concuerdan en establecer una situación crítica para la sobrevivencia de esta especie en Chile, condición preocupante, por la casi nula intervención de la comunidad entomológica y de los actores gubernamentales sobre su continuidad en el futuro.

El índice SUMIN que categoriza a $C$. granti como especie Rara, sobrevalora variables relacionadas con patrones de distribución, grado de amenaza y protección en unidades de conservación; sin embargo, variables importantes para la definición de rareza como son la abundancia y singularidad de una especie, resultan de poca importancia. Este índice además no evalúa a todas las variables en forma equitativa, ya que sus valores máximos fluctúan entre 1 a 5 en variables de poca y de mayor importancia, como ocurre con la abundancia y la distribución nacional, respectivamente. Por todo esto, creemos que este índice no sería adecuado para la categorización de insectos en conservación.

El índice CPI por el contrario, evalúa todas las variables con igual importancia y define a especies con mayor riesgo de extinción, como aquellas especies grandes, habitantes de un menor número de ecorregiones, con rango de distribución restringido, menor abundancia, endemismo, singularidad, sometida a mayor extracción antrópica y con un menor grado de protección. Este índice identifica a $C$. granti como especie Vulnerable. Para CONAMA (2005), esta categorización indicaría que "la especie enfrenta un riesgo grande de extinción, no pudiendo, sin embargo, ser catalogada como especie En Peligro". Se agrega a esto la distribución restringida que también existe en Argentina (en las provincias de Neuquén, Río Negro, Chubut) que impide una eventual recolonización o intercambio de las poblaciones siendo esta una situación crítica para el establecimiento de esta especie en el futuro.

Ambos índices no son concordantes en la categorización de $C$. granti, debido a que difieren en sus categorías de conservación y en la evaluación de sus variables. Por este mismo motivo, las estrategias de conservación, sostienen que una evaluación equitativa de cada variable resulta ser más eficaz para la categorización de especies. Esta situación es aplicable en la evaluación del índice CPI, por lo que concluimos que $C$. granti presenta actualmente en Chile un estado de conservación Vulnerable pudiendo este índice ser aplicable a otras especies de insectos.

Es pertinente destacar que algunas de las variables evaluadas por SUMIN y CPI, son difíciles de determinar para coleópteros, al carecer de información completa sobre aspectos ecológicos, biogeográficos y biológicos. Dentro de estas variables se encuentran el Potencial reproductivo de la especie, la masa corporal y su abundancia relativa, variables que en un futuro pueden ser reemplazadas por características relevantes en insectos como lo fue en este caso, el reemplazo de la variable masa corporal del índice CPI por medidas de longitud corporal. Sin embargo, con esta propuesta es posible sentar las bases de la utilización del índice CPI en futuros estudios en conservación de insectos en Chile. 


\section{AGRADECIMIENTOS}

Agradecemos a Mario Elgueta (Museo Nacional de Historia Natural, Chile), Jorge Artigas (Museo de Zoología, Universidad de Concepción, Chile), Jaime Solervicens (Instituto de Entomología Universidad Metropolitana de Ciencias de la Educación, Chile), Sergio Rothman, Marcos Beeche y José Mondaca (Servicio Agrícola y Ganadero, Chile), Federico Ocampo y Gustavo Flores (Instituto Argentino de Investigaciones de Zonas Áridas, Argentina), James Boone y Alfred Newton (Field Museum, EE.UU.), Apóstol Tenorio y Jorge Poblete (Corporación Nacional Forestal, Chile), Roget Alba (Parque Oncol, Valdivia, Chile). A Patricio Pliscoff y Ernesto Teneb por la facilitación de material digital. A los colegas Biol. Johara Bourke, Gustavo Valenzuela, Raúl Briones, Jonathan Guzmán-Sandoval, Christian Muñoz por la ayuda en terreno y aportes en este manuscrito. Finalmente a los revisores anónimos por sus comentarios. Este trabajo fue financiado por el Proyecto DIUC No 205.113.066-1.0 de la Dirección de Investigación de la Universidad de Concepción.

\section{LITERATURA CITADA}

ARROYO MTK, JJ ARMESTO \& FA SQUEO (2008) Conservación de especies amenazadas a nivel global y regional. En: Squeo FA, G Arancio \& J Gutiérrez (eds) Libro rojo de la flora nativa y de los sitios prioritarios para su conservación, Región de Atacama: 3-12. Ediciones Universidad de La Serena, Chile

ARZAMENDIA V \& A GIRAUDO (2004) Usando patrones de biodiversidad para la evaluación y diseño de áreas protegidas: las serpientes de la provincia de Santa Fe (Argentina) como ejemplo. Revista Chilena de Historia Natural 77: 335-348.

BOSSART J \& CH CARLTON (2002) Insect conservation in America: Status and perspectives. American Entomologist 48: 82-92.

CHRISTIE MI (1984) Determinación de prioridades conservacionistas para la fauna de vertebrados patagónicos. Revista del Museo Argentino de Ciencias Naturales, Buenos Aires. Zoología 13: 535-539.

CONAMA (2003) Estrategia nacional de biodiversidad. Comisión Nacional del Medio Ambiente, Chile. URL: http://www.conama.cl (accedido Mayo 12, 2009).

CONAMA (2005) Reglamento para la clasificación de especies silvestres en Chile. Comisión Nacional del Medio Ambiente, Chile. URL: http:// www.conama.cl/clasificacionespecies (accedido Mayo 12, 2009)

COFRÉ H \& P MARQUET (1999) Conservation status, rarity, and geographic priorities for conservation of Chilean mammals: an assessment. Biological Conservation 88: 53-68.

DE GRAMMONT P \& A CUARÓN (2006) An evaluation of threatened species categorization systems used on the American Continent. Conservation Biology 20: $14-27$.

DÍAZ-PÁEZ H \& JC ORTIZ (2003) Evaluación del estado de conservación de los anfibios en Chile. Revista Chilena de Historia Natural 76: 509-525.

DOMÍNGUEZ MC, S ROIG-JUÑENT, JJ TASSIN, F OCAMPO \& G FLORES (2006) Areas of endemism of the Patagonian steppe: An approach based on insect distributional patterns using endemicity analysis. Journal of Biogeography 33: 1527-1537.

EYRE MD \& P RUSHTON (1989) Quantification of conservation criteria using invertebrates. Journal of Applied Ecology 26: 159-171.

FLEISHMAN E, D MURPHY \& P BRUSSARD (2000) A new method for selection of umbrella species for conservation planning. Ecological Applications 10: 569-579.

GLADE A (ed) (1988) Libro rojo de los vertebrados terrestres de Chile. Actas del Simposio «Estado de Conservación de los Vertebrados Terrestres de Chile». CONAF ediciones, Santiago, Chile. 67 pp.

IRIARTE J, P FEINSINGER \& F JAKSIC (1997) Trends in wildlife use and trade in Chile. Biological Conservation 81: 9-20.

IUCN (2001) Conservación mundial. Vigilando a las especies amenazadas. La lista roja de la IUCN, International Union for Conservation of Nature. Boletín de la IUCN 3: 24.

JIMÉNEZ M (1999) Evaluación del estado de conservación de las aves de Humedal de la Región del Biobío. Unidad de Recursos Naturales Editores. Comisión Nacional del Medio Ambiente, Región del Biobío, Chile. 38 pp.

KELLERT S (1993) Values and perceptions of invertebrates. Conservation Biology 7: 845-855.

LUEBERT F \& P PLISCOFF (2006) Sinopsis bioclimática y vegetacional de Chile. Primera edición, Editorial Universitaria, Chile. 316 pp.

MORRONE JJ (2006) Biogeographic areas and transition zones of Latin America and the caribbean islands based on panbiogeographic and cladistic analyses of the entomofauna. Annual Review of Entomology 51: 467-94

MYERS N, R MITTERMEIER, C MITTERMEIER, G DA FONSECA \& J KENT (2000) Biodiversity hotspots for conservation priorities. Nature 403: 853-858.

NAVARRETE M (1992) Gran atlas de Chile y del universo. Ediciones Occidente SA, Chile. 100 pp.

NUMHAUSER J (1981) Perspectiva histórica de los cambios sinonímicos en las especies del género Chiasognathus Stephens (Ins. Coleoptera. Lucanidae). Revista Chilena de Entomología 11: 17-28.

PELLET P, E UGARTE, E OSORIO \& F HERRERA (2005) Conservación de la biodiversidad en Chile, ¿legalmente suficiente? La necesidad de cartografiar la ley antes de decidir. Revista Chilena de Historia Natural 78: 125-141.

POVILITIS A (1998) Characteristics and conservation of a fragmented population of huemul Hippocamelus bisulcus in central Chile. Biological Conservation 86: $97-104$

PRIMACK R, R ROZZI, P FEINSINGER, R DIRZO \& F MASSARDO (eds) (2001) Fundamentos de conservación biológica, perspectivas 
latinoamericanas. Primera edición. Fondo de Cultura Económica, México DF. 497 pp.

RABINOWITZ D (1981) Seven forms of rarity. En: Synge $\mathrm{H}$ (ed) The biological aspects of rare plant conservation: 205-212. John Wiley and Sons, Chichester, NH.

RECA A, C ÚBEDA \& D GRIGERA (1994) Conservación de la fauna de tetrápodos I. Un índice para su evaluación. Mastozoología Neotropical 1: 17-28.

REDAK R (2000) Arthropods and multispecies habitat conservation plans: Are we missing something? Environmental Management 26: 97-107.

REFSETH D (1980) Ecological analyses of carabid communities-potential use in biological classification for nature conservation. Biological Conservation 17: 131-141

ROIG-JUÑENT S, J CRISCI, P POSADAS \& S LAGOS (2002) Áreas de distribución y endemismo en zonas continentales. En: Costa C, S Vanin, J Lobos \& A Melic (eds) Proyecto Red Iberoamericana de Biogeografía y Entomología Sistemática: 247-266. Monografías del Tercer Milenio, volumen 2. Sociedad Entomológica Aragonesa (SEA), Zaragoza.

ROJAS M (1992) The species problem and conservation: What are we protecting? Conservation Biology 6: 170-178.

ROZZI R, R PRIMACK, P FEINSINGER, R DIRZO \& F MASSARDO (2001) ¿Qué es la biología de la conservación? En: Primack R, R Ruiz, P Feinsinger, R Dirzo \& F Massardo (eds) Fundamentos de conservación biológica, perspectivas latinoamericanas: 35-43. Primera edición, Fondo de Cultura Económica, México DF.

SAG (1998) Cartilla de caza. Primera edición. Servicio Agrícola y Ganadero, Chile. Departamento de protección de los recursos naturales renovables editores, Chile. 84 pp.

SAMWAYS M (2001) Los invertebrados de África Meridional: cuidando a la multitud. Boletín de la UICN, Conservación mundial 3: 25.
SCHWARTZ M (1999) Choosing the appropriate scale of reserves for conservation. Annuals Review Ecology and Systematic 30: 83-108.

SIMONETTI J (1997) Biodiversity and a taxonomy of Chilean taxonomists. Biodiversity and Conservation 6: 633-637.

SMITH R \& T SMITH (2001) Ecología. Cuarta edición. Pearson educación S.A. Madrid. 664 pp.

SQUEO F, G ARANCIO, L CAVIERES, M MUÑOZ \& C MARTICORENA (2001) Detalle de los criterios metodológicos y listado de especies analizadas con los valores de los índices utilizados para establecer su estado de conservación a nivel regional. En: Squeo F, G Arancio \& J Gutiérrez (eds) Libro rojo de la flora nativa y de los sitios prioritarios para su conservación: Región de Coquimbo: 323-352. Ediciones Universidad de La Serena, Chile.

STEPHENS JF (1831) Description of Chiasognathus grantii, a new Lucanideous insect forming the type of an undescribed genus, together with some brief remarks upon its structure and affinities. In a letter addressed to one of the secretaries. Transactions Cambridge Philosophical Society 4: 209-217.

THOMAS CD \& HC MALLORIE (1985) Rarity, species richness and conservation: Butterflies of the Atlas Mountains in Morocco. Biological Conservation 33: 95-117.

ÚBEDA C, D GRIGERA \& A RECA (1994) Conservación de la fauna de tetrápodos II. Estado de conservación de los mamíferos del Parque y Reserva Nacional Nahuel Huapi. Mastozoología Neotropical 1: 29-44.

VERDÚ JR \& GALANTE (eds) (2005) Libro rojo de los invertebrados de España. Dirección General de Conservación de la Nsaturaleza, Madrid, España (en línea) URL: http://carn.ua.es/CIBIO/es/lrie/ lrie.html (accedido Mayo 12, 2009).

WHITTAKER R, M ARAÚJO, P JEPSON, R LADLE, J WATSON \& K WILLIS (2005) Conservation biogeography: Assessment and prospect. Diversity and Distributions 11: 3-23. 